\title{
Adaptive inverse filtering to equalize and linearize a loudspeaker response
}

\author{
Markus Gross* and Masato Miyoshi** \\ *Fachhochschule Regensburg Fachbereich Elektrotechnik, Seybothstrasse 2, 93053 Regensburg, Germany \\ **NTT Human Interface Laboratories, 3-9-11, Midori-cho Musashino, Tokyo, 180-8585 Japan \\ (Received 19 February 1998)
}

Keywords: Adaptive filter, Equalizer, Inverse, Loudspeaker, Nonminimum phase PACS number: 43. 38. Md

\section{Introduction}

The adaptive inverse filtering proposed by Widrow and Walach, ${ }^{1)}$ as shown in Fig. 1, seems effective and elegant in equalizing a transfer function of a loudspeaker (hereafter called an acoustic plant) to be flatmagnitude and linear-phase, since the method does not need to pre-measure the plant. In Fig. 1, the inverse of the plant $z^{-\Delta} G(z)$ is set to the controller $C(z)$ to equalize the plant, after the convergence of the adaptive inverse filter $H(z)$ :

$$
C(z) \cdot z^{-\Delta} G(z)=H(z) \cdot z^{-\Delta} G(z)=z^{-(\Delta+m)},
$$

where the delay of $m(m=1,2, \cdots)$ samples is needed when $G(z)$ is nonminimum phase. For conducting the continuous equalization in a reverberant room with an input signal $i(k)$ like music, however, this method should be modified by considering;

(a) "Ringing" arises from the excessive amplification of the input by $C(z)(=H(z))$ at certain frequencies corresponding to nonminimum phase zeros of $G(z)$, and

(b) The sound information pieces whose frequencies are eliminated by $C(z)$ are unrecoverable.

The first problem can be avoided by adding a pseudo random noise to the input of the adaptive inverse filter $H(z)$. However, the noise level should be set carefully to avoid degradation of the inverse filtering accuracy. Increasing the number of delay samples $m$ is also effective, however, the problem caused by the nonminimum phase zeros very close to the unit circle on the $z$-plane does not seem to sufficiently be solved.

As to the second problem, a typical case may be seen when using a music signal for the input $i(k)$, where a component covering a certain frequency range is first supplied for a while, and next, another component covering another frequency range, and so on. In this case, the frequency response of the adaptive inverse filter $H(z)$ sometimes becomes zero for the second frequency range during the first period due to, for example, thermal noise and/or background noise mixed in the input of the filter. If this $H(z)$ is set to the controller $C(z)$ before the second period begins, the second component will not be supplied any more.

This short report proposes a few possible modifications to the conventional method in order to solve these problems.

\section{Proposed modifications}

The proposed modifications are shown in Fig. 2. Here, a band elimination filter $(B E F)$ is added for attenuating the input signal components that will be excessively amplified by the controller $C(z)$, a duplicate of the adaptive inverse filter $H(z)$. Two bypass circuits featured by the delay units of $z^{-m}$ are also added for the abovementioned second problem. Below, the functions of these newly introduced devices will precisely be explained by referring the time points in Fig. 3 which shows the changes in the power ratio of the estimation error $e(k)$ to the reference $d(k)$ in a computer simulation. The parameters used for this simulation are also given in Table 1.

The frequency range of an input random noise $i(k)$ is restricted up to $12 \mathrm{kHz}$ until time point $B$. During this period, the adaptive inverse filter $H(z)$ is iteratively calculated $^{2)}$ and a converged transfer function is set to the controller $C(z)$ at time point $A$. Looking at the frequency characteristics of $C(z)(=H(z))$ shown in Fig. 4, the excessive amplification at around 0,6 and 11 $\mathrm{kHz}$, and the attenuation of the frequency range above $12 \mathrm{kHz}$ can be clarified.

At time point $A$, the $B E F$ is simultaneously designed to cancel out such excessive amplification at the controller $C(z)$ by using the power spectral information of the estimation error $e(k)$ to the reference $d(k)$, namely $\{e(f)\}^{2} /\{d(f)\}^{2}(f$ : frequency). This idea comes from the observation that the attenuation of the estimation error is very low at those undesirable frequencies corresponding to nonminimum phase zeros in comparison with that at other frequencies when a sufficient number of taps is given to the controller $C(z){ }^{3)}$ Three peaks at around 0,6 and $11 \mathrm{kHz}$ shown in Fig. 5, therefore, are considered such undesirable frequencies in our method. The ridge above $12 \mathrm{kHz}$ is apparently caused by the frequency range limitation on the input $i(k)$. However, this frequency range is also considered undesired by the above-mentioned idea concerning $\{e(f)\}^{2} /\{d(f)\}^{2}$. The magnitude-frequency response of the $B E F$ is first calculated to be zeros at these frequencies, $0,6,11$ and $12 \mathrm{kHz}$, and ones $(=1)$ at other frequencies. Then, this frequency response is converted to an impulse response of a given length by using the Inverse Fast Fourier Transform and a Kaiser window ${ }^{4}$ so that the $B E F$ becomes a linear-phase FIR filter. ${ }^{5)}$ The $B E F$ attenuates the input $i(k)$ at those undesired 
frequencies, 0,6 and $11 \mathrm{kHz}$, in order that $i(k)$ will not

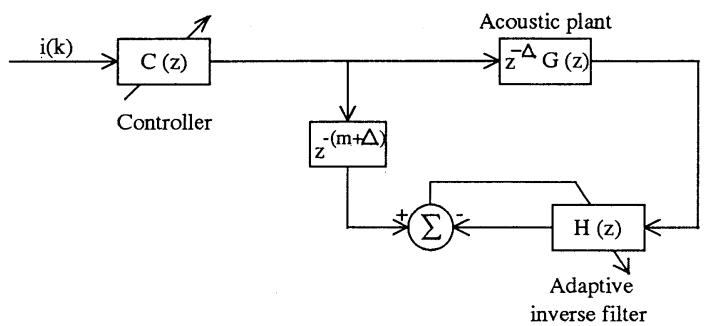

become so large after processing by the controller $C(z)$, and, as a result, the "ringing" can be avoided. Figure 6 shows the consequent frequency characteristics of the

Table 1 Parameters in computer simulation.

\begin{tabular}{lc}
\hline & No. of taps used \\
\hline Sampling frequency & $48 \mathrm{kHz}$ \\
Acoustic plant & 750 with the delay $\Delta$ of 350 \\
Adaptive filter & 800 \\
Delay $m$ & 400 \\
BEF and LPF & 256 with the delay of 128 \\
\hline
\end{tabular}

Fig. 1 Adaptive inverse filtering by Widrow and Walach.

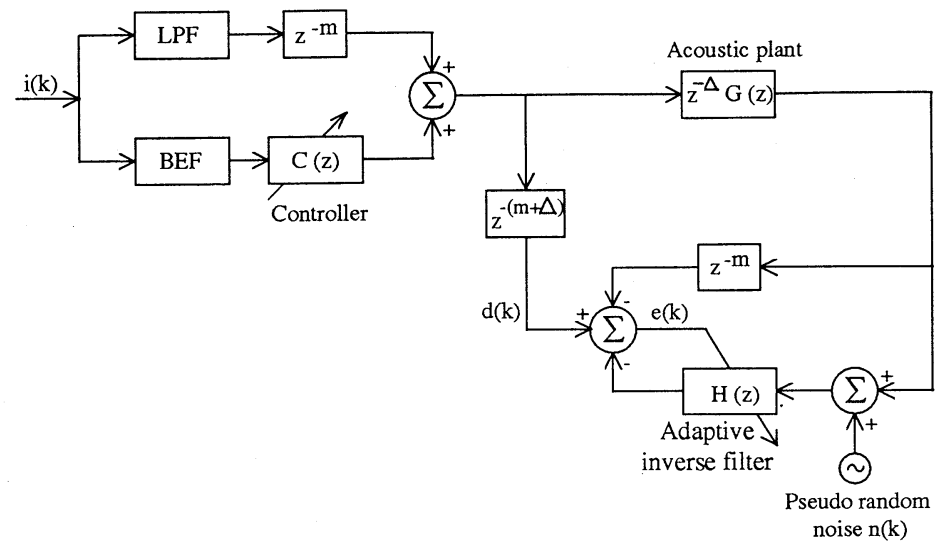

Fig. 2 Modified adaptive inverse filtering.

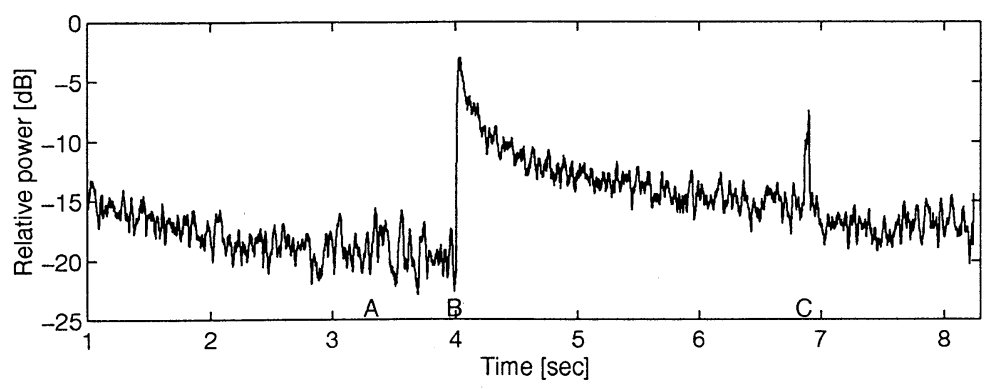

Fig. 3 Power ratio of estimation error $e(k)$ to reference $d(k) . A, B, C$, time points.

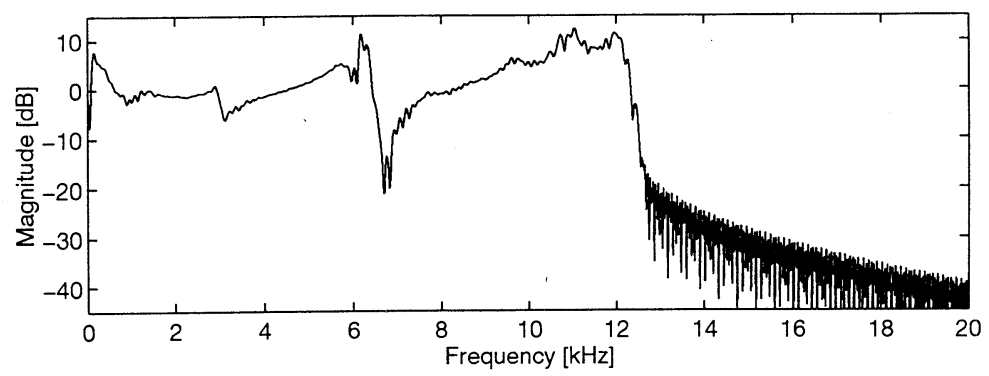

Fig. 4 Frequency response of controller $C(z)$. 


\section{GROSS and M. MIYOSHI : LOUDSPEAKER EQUALIZATION \& LINEARIZATION}

(a)

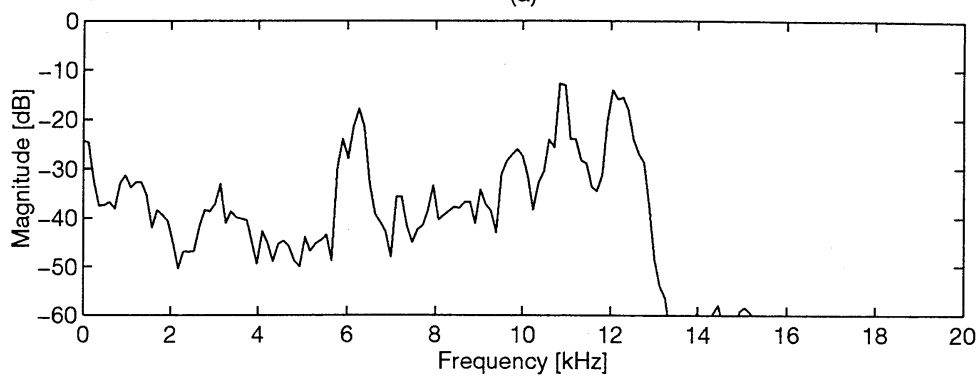

(b)

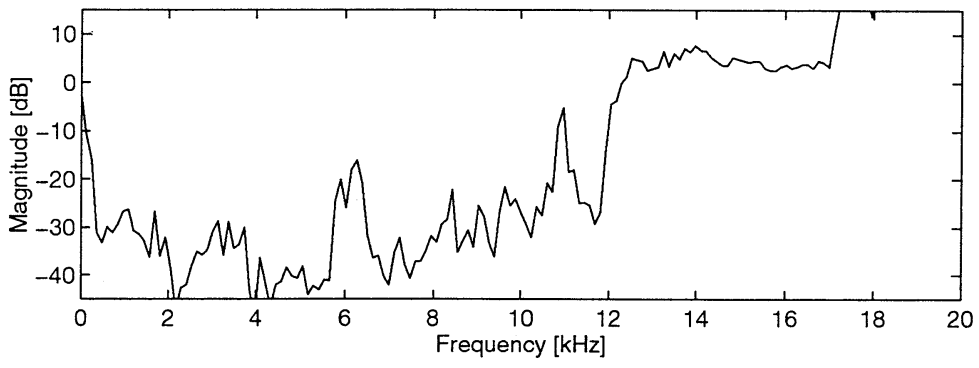

Fig. 5 Power spectrum of estimation error $e(k)$ at time point $A$. (a) e(k), (b) $e(k)$ to $d(k)$.

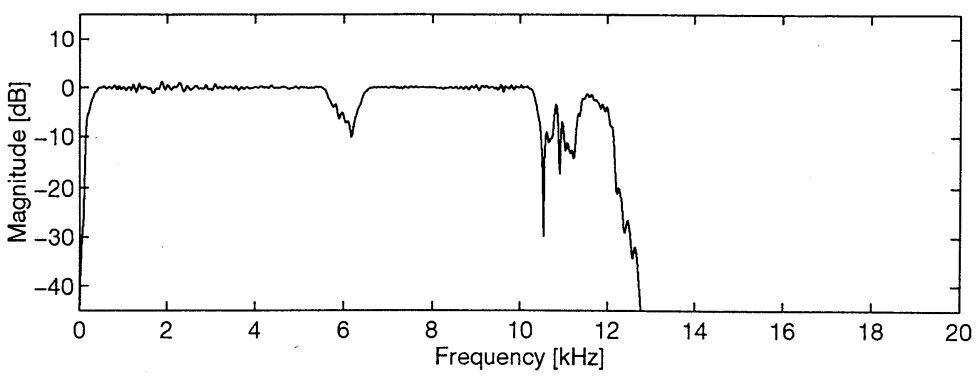

Fig. 6 Frequency response of equalized plant output before time point $B$.

equalized plant output just before time point $B$, where the plant $z^{-4} G(z)$ is equalized up to $12 \mathrm{kHz}$.

At time point $B$, the frequency range of the input $i(k)$ is expanded up to $16 \mathrm{kHz}$. Here, the first bypass circuit over the $B E F$ and controller $C(z)$ enables the adaptive inverse filter $H(z)$ to track this frequency expansion, since the spectral information above $12 \mathrm{kHz}$ is not supplied to $H(z)$ via the $B E F$ and $C(z)$. Below $12 \mathrm{kHz}$, on the other hand, the first bypass circuit, $B E F$ and $C(z)$ cooperatively synthesize a signal to maintain the equalization of the acoustic plant $z^{-4} G(z)$ as well as continuously calculate $H(z)$. These faculties are obtained, through the period up to time point $A$, by the functions of the second bypass circuit over $H(z)$ and a pseudo random noise $n(k) ; H(z)$ converges to zero above $12 \mathrm{kHz}$, since $n(k)$ is added to the input of $H(z)$ but both the reference $d(k)$ and the output of the second bypass circuit are zeros, and below $12 \mathrm{kHz}$, $H(z)$ is calculated so as to make the summation of the output signals of the second bypass circuit and $H(k)$ a good replica of $d(k)$. The conventional method shown in Fig. 1, however, does not have this kind of tracking ability. Figure 7 shows the frequency characteristics of the equalized plant output just after the second setting of $H(z)$ to the controller $C(z)$ at time point $C$. The $B E F$ is also renewed with the abovementioned design procedure. The acoustic plant $z^{-4} G(z)$ is successfully equalized up to $16 \mathrm{kHz}$.

\section{Summary}

A few modifications of conventional adaptive inverse filtering are proposed in order to equalize a loudspeaker response to be flat-magnitude and linear-phase with the suppression of "ringing" and with ability to track the spectral information changes in the input. Computer simulation demonstrated the validity of the proposed modifications. 
(a)

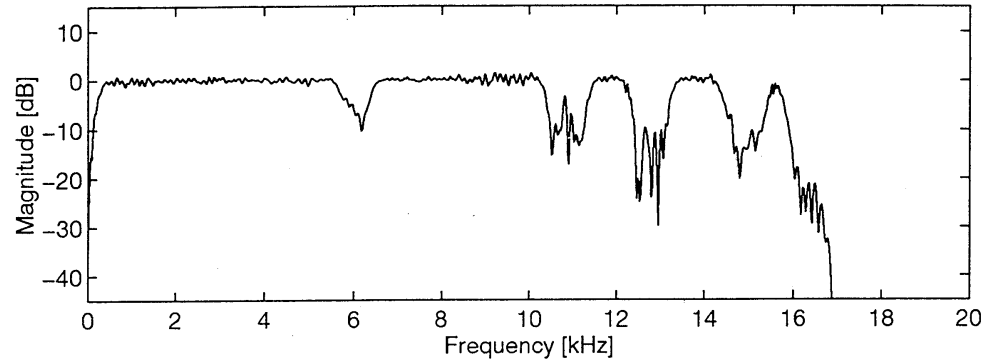

(b)

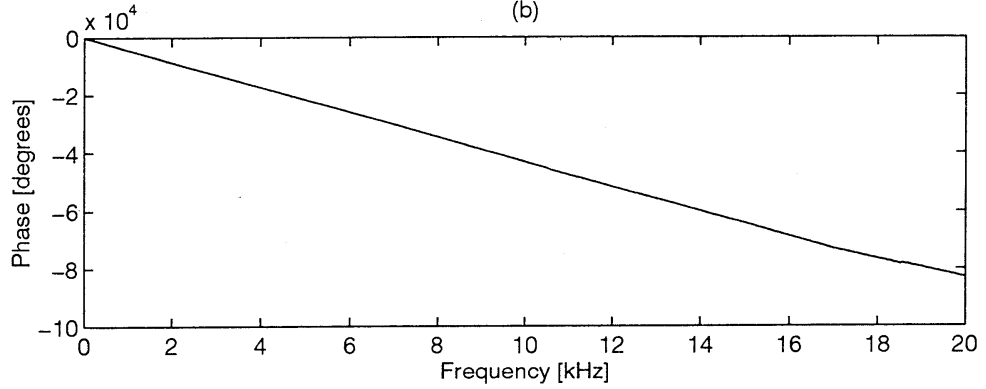

Fig. 7 Frequency response of equalized plant after time point $C$.

(a) Magnitude, (b) Phase.

(a)

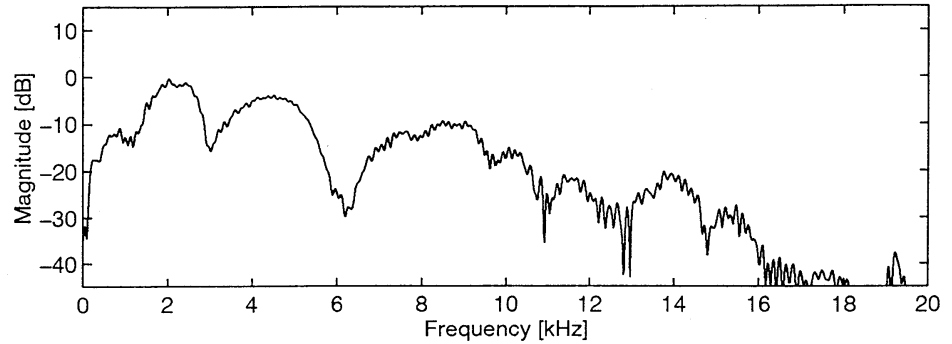

(b)

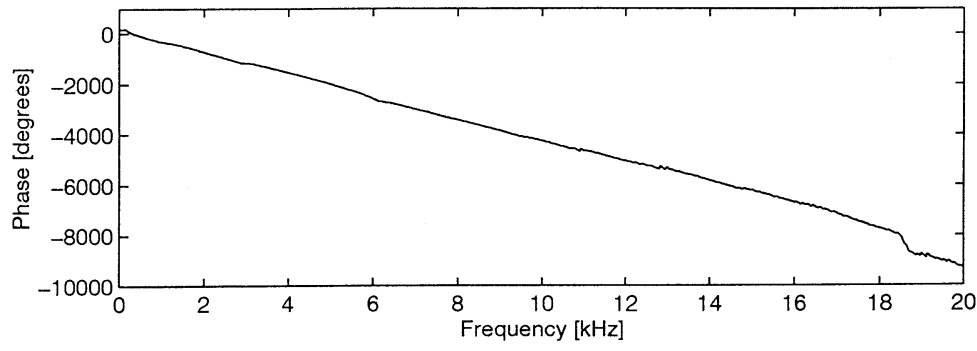

Fig. 8 Frequency response of original acoustic plant $z^{\Delta} G(z)$.

(a) Magnitude, (b) Phase.

References

1) B. Widrow and E. Walach, "Adaptive signal processing for adaptive control,” Proc. ICASSP84, 21. 1. 1-4 (1984).

2) S. Haykin, Adaptive Filter Theory (Prentice-Hall, Englewood Cliffs, NJ, 1991), pp. 352-356.

3) M. Miyoshi and Y. Kaneda, "Inverse filtering of room acoustics," IEEE Trans. Acoust. Speech Signal Process. 36(2), 145-152 (1988).

4) J. V. Candy, Signal Processing-The Modern Approach-(McGraw-Hill, New York, 1988), p. 47.

5) A. V. Oppenheim and R. W. Shafer, Digital Signal Processing (Prentice-Hall, Englewood Cliffs, NJ, 1975), pp. 251-255. 